\title{
The extracellular matrix of the cuticle of Gordius panigettensis (Gordioiidae, Nematomorpha): observations by TEM, SEM and AFM
}

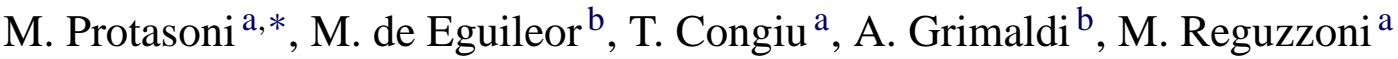 \\ a Laboratory of Human Morphology, University of Insubria, via Monte Generoso 71, 21100 Varese, Italy \\ ${ }^{\mathrm{b}}$ Department of Structural And Functional Biology, University of Insubria, via Dunant 5, 21100 Varese, Italy
}

Received 19 March 2003; received in revised form 6 May 2003; accepted 21 May 2003

\begin{abstract}
The cuticle of Gordius panigettensis (Sciacchitano, 1955) was studied by scanning electron microscopy (SEM), transmission electron microscopy (TEM) and atomic force microscopy (AFM).

The cuticle is composed of 30-50 compact layers. The number of the layers is higher in the central part of the animal's body and decreases at the extremities. Each layer is composed of parallel tightly packed fibres approximately 640 nm in diameter and of indefinite length. The fibres run strictly parallel within each layer, while in adjoining layers they run at a variable angle from $45^{\circ}$ in the central body to $90^{\circ}$ in the extremities. Each fibre shows a barely detectable filamentous inner structure and is enveloped in a thin highly regular net formed by hexagonal meshes. Our results suggested that these fibres should be proteinaceous although non-collagenous. Thinner radial fibres run among the large fibres and across all the layers and span the whole thickness of the cuticle from the epithelial layer located deep underneath the large fibres up to the epicuticle on the external surface of the animal.
\end{abstract}

(C) 2003 Elsevier Ltd. All rights reserved.

Keywords: Gordius panigettensis; Extracellular matrix

\section{Introduction}

Many small soft-bodied invertebrates maintain their body shape by turning themselves into a pressure vessel where internal hydrostatic pressure is contained and withstood by a tough external cuticle, not unlike the walls of a tyre resisting inflation pressure. For such a structure to function correctly, the vessel walls must be flexible but very strong and stiff, so that any increase in inner pressure is counterbalanced by a sharp increase in the tangential tension in the cuticle. Beyond its obvious involvement in locomotion, the cuticle is also responsible for protecting the inner organs from mechanical and chemical attacks. In parasite forms, it also constitutes the interface with the host tissues, so it must also be chemically stable. The cuticle of Nematodes, Nematomorphs and, in particular, Annelids has therefore been the subject of several structural and ultrastructural studies (Swanson, 1970; Eakin and Brandenburger, 1974; Lanzavecchia et al., 1979; Cham

${ }^{*}$ Corresponding author. Tel: +39-0332-264847; fax: +39-0332-263324.

E-mail address: marina.protasoni@libero.it (M. Protasoni). et al., 1983; Bresciani, 1991; Politz and Philipp, 1992; Brivio et al., 2000).

The present research studied the cuticle of Gordius panigettensis by scanning electron microscopy (SEM), transmission electron microscopy (TEM) and atomic force microscopy (AFM) to investigate and describe the different elements which constitute the cuticle and contribute to maintain its critical architecture. Maceration techniques and histochemical reactions were also applied prior to the SEM and TEM, respectively, to gain different viewpoints and to shed light on the cuticle ultrastructure.

\section{Materials and methods}

Adult G. panigettensis worms were collected from the Serio river (Bergamo, Italy).

Cuticle samples were isolated from the worm body by manual stripping and were easily freed of the epithelium under a stereomicroscope. The specimens were then divided into five groups, which underwent different treatments as follows. 


\subsection{Scanning electron microscopy}

The isolated cuticles were cut into pieces of $2 \mathrm{~mm}$ and fixed in $0.25 \%$ glutaraldehyde and $0.25 \%$ paraformaldehyde in $0.1 \mathrm{M} \mathrm{Na}$-cacodylate buffer for $20 \mathrm{~min}$ at room temperature, then rinsed in cold cacodylate buffer and dehydrated in graded ethanol and hexamethyldisilazane. The specimens were then mounted on aluminium stubs, sputter-coated with gold in an Emitech K550 apparatus and observed in a Philips XL-30 SEM-FEG scanning electron microscope operated at $10 \mathrm{kV}$.

\section{2. $\mathrm{NaOH}$ maceration}

The specimens were fixed for five days with $0.25 \%$ glutaraldehyde and $0.25 \%$ paraformaldehyde in $0.1 \mathrm{M}$ Na-cacodylate buffer ( $\mathrm{pH} 7.2)$ and then cut with a razor blade into $0.5 \mathrm{~mm}$ thick slices, rapidly washed in distilled water and immersed in $1 \mathrm{~N} \mathrm{NaOH}$ for three days at room temperature. The slices were then washed in distilled water for three days at room temperature, dehydrated in graded ethanol and critical-point dried in an Emitech K850 CPD apparatus. The slices were then mounted, coated and observed as above.

\subsection{Osmic maceration}

The specimens were fixed in $0.25 \%$ glutaraldehyde and $0.25 \%$ paraformaldehyde in $0.1 \mathrm{M} \mathrm{Na-cacodylate}$ buffer $(\mathrm{pH}$ 7.2) for $20 \mathrm{~min}$ at room temperature. After a first reduction in size of $1 \mathrm{~mm}^{3}$, the specimens were washed in phosphate-buffered saline (PBS, $\mathrm{pH}$ 7.2) and then post-fixed in a solution of $1 \%$ osmium tetroxide and $1.25 \%$ potassium ferrocyanide for $2 \mathrm{~h}$. The specimens underwent a further reduction in slices $0.2 \mathrm{~mm}$ thick, and a second post-fixation in $1 \%$ osmium tetroxide and $1.25 \%$ potassium ferrocyanide for $1 \mathrm{~h}$. The slices were washed in PBS and immersed in $0.1 \%$ osmium tetroxide in PBS for $48 \mathrm{~h}$. They were then dehydrated in graded ethanol and critical-point dried, mounted, coated and observed as above.

\subsection{Histochemistry}

The same specimens treated in osmic maceration were dehydrated in graded ethanol and propylene oxide and embedded in EPON 812. Ultrathin sections were cut with a diamond knife, collected on 200 mesh copper grids and subjected to the Thiery reaction (Thiery, 1967). The grids were then observed on a JEOL 1010 transmission electron microscope operated at $80 \mathrm{kV}$.

\subsection{Atomic force microscopy}

The isolated cuticles were fixed in $0.25 \%$ glutaraldehyde and $0.25 \%$ paraformaldehyde in $0.1 \mathrm{M} \mathrm{Na}$-cacodylate buffer for $20 \mathrm{~min}$ at room temperature, then rinsed in cold cacodylate buffer and dehydrated in graded ethanol and hexamethyldisilazane. Specimens were then observed in tapping-mode atomic force microscopy (TMAFM) on a Digital Instruments Nanoscope III multi-mode microscope, fitted with Nanosensors SSS-NCH silicon probes (force constant $30-60 \mathrm{~N} / \mathrm{m}$, resonance frequency $300-350 \mathrm{kHz}$ ). All observations were carried out in air at a scan rate of approximately $2 \mathrm{~Hz}$.

To enhance the detail of the TMAFM images without compromising their accuracy and linearity, TMAFM files were rendered in three-dimension by photo-realistic ray-tracing rendering software (POV-RAY version 3.0, http://www.povray.org).

\section{Results}

A careful peeling of the cuticle disclosed the internal structure of each layer and the relationship between the fibres within each layer and among adjoining layers (Fig. 1).

The fibres had a regular constant diameter of $640 \mathrm{~nm}$ and were arranged strictly parallel to each other. Defects and malformations, such as bifurcated fibre, were only occasionally observed (Fig. 2). No periodic banding was noted in any of the specimens. Each fibre appeared entirely wrapped in a highly regular net formed by hexagonal meshes. Sometimes, in the process of peeling the layers, part of the covering remained stuck on the contiguous layers where it maintained a cast of the fibres removed. Thin fibres are interposed perpendicularly between the large fibres (Figs. 1 and 3).

All the fibres and coverings remained intact after the prolonged $1 \mathrm{~N} \mathrm{NaOH}$ maceration, thus indicating an exceptional resistance of the major components of the cuticle. The fibres, roughly, quadrangular in section, again appeared parallel and their hexagonal mesh covering was well evident (Fig. 4).

Thin radial fibres, running perpendicular to the major axis of the animal, were well evident on the surface of the large fibres often grouped in small ribbons periodically strewn between the large fibres. The distance between the ribbons was regular and corresponded to the diameter of the large fibres. Some slender single filaments ran between the ribbons, sometimes parallel, sometimes perpendicular, to interconnect adjacent ribbons (Fig. 4).

The loosening of the elements composing the fibres, due to the $\mathrm{NaOH}$ maceration, revealed the internal structure of the large fibres, where thin filaments formed flat laminae which, in turn, were stacked together to form large fibres (Fig. 5).

A short osmic maceration partially dissolved the fibres and left the perifibrillar covering intact. Some slender tubular structures appeared to run perpendicularly in the space between adjacent fibres, in the same arrangement and 

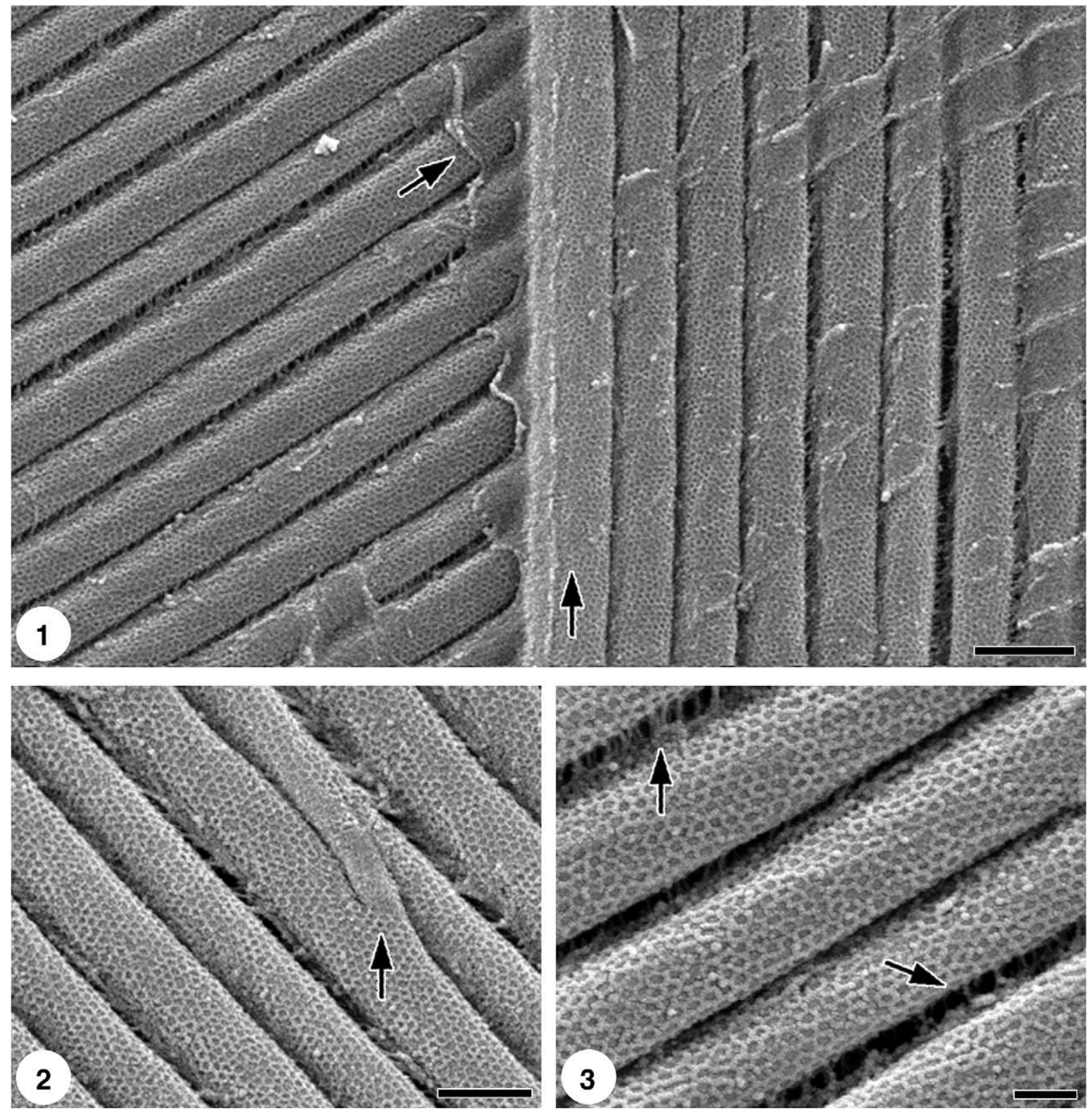

Fig. 1. SEM. The fibres of two contiguous layers of the cuticle appear strictly parallel within each layer, while an angle of approximately $45^{\circ}$ appears between contiguous layers. A sort of ordered sticky covering is easily recognisable on the surface of the fibres. During peeling, the covering can remain on the layer below, still showing the cast of the fibres above (arrows). Scale bar $1 \mu \mathrm{m}$. Fig. 2. SEM. A rare bifurcated fibre (arrow). Scale bar $1 \mu \mathrm{m}$. Fig. 3. SEM. A regular hexagonal mesh network envelops the fibres. Thin fibres (arrows) join the coverings of contiguous large fibres. Scale bar $500 \mathrm{~nm}$.

localisation of the ribbons as the thin fibres previously described (Fig. 6).

By contrast, a prolonged osmic maceration completely dissolved the fibres but again left their perifibrillar coverings intact. The complete removal of the fibres suggested that they are proteinaceous, whereas their perifibrillar covering has a different composition. The covering of each large fibre remained as individual hollow shells, approximately rectangular in section, closely packed side by side. A narrow space between the sides of contiguous envelopes was readily detectable. Perifibrillar shells were still parallel to each other within the same layer and were oriented from 45 to $90^{\circ}$, following the orientation of the respective fibres, in contiguous layers (Fig. 7).

The complete removal of the fibres granted us access to the internal structure of the perifibrillar envelope. The walls still showed a highly regular network formed by hexagonal mesh, perfectly comparable to the external wall of the envelope observed on the fibre surface with classical SEM (Fig. 7, insert). Under the TEM, silver deposits from the Thiery reaction were detected on the perifibrillar covering, whereas both the large fibres and the perpendicular filaments 

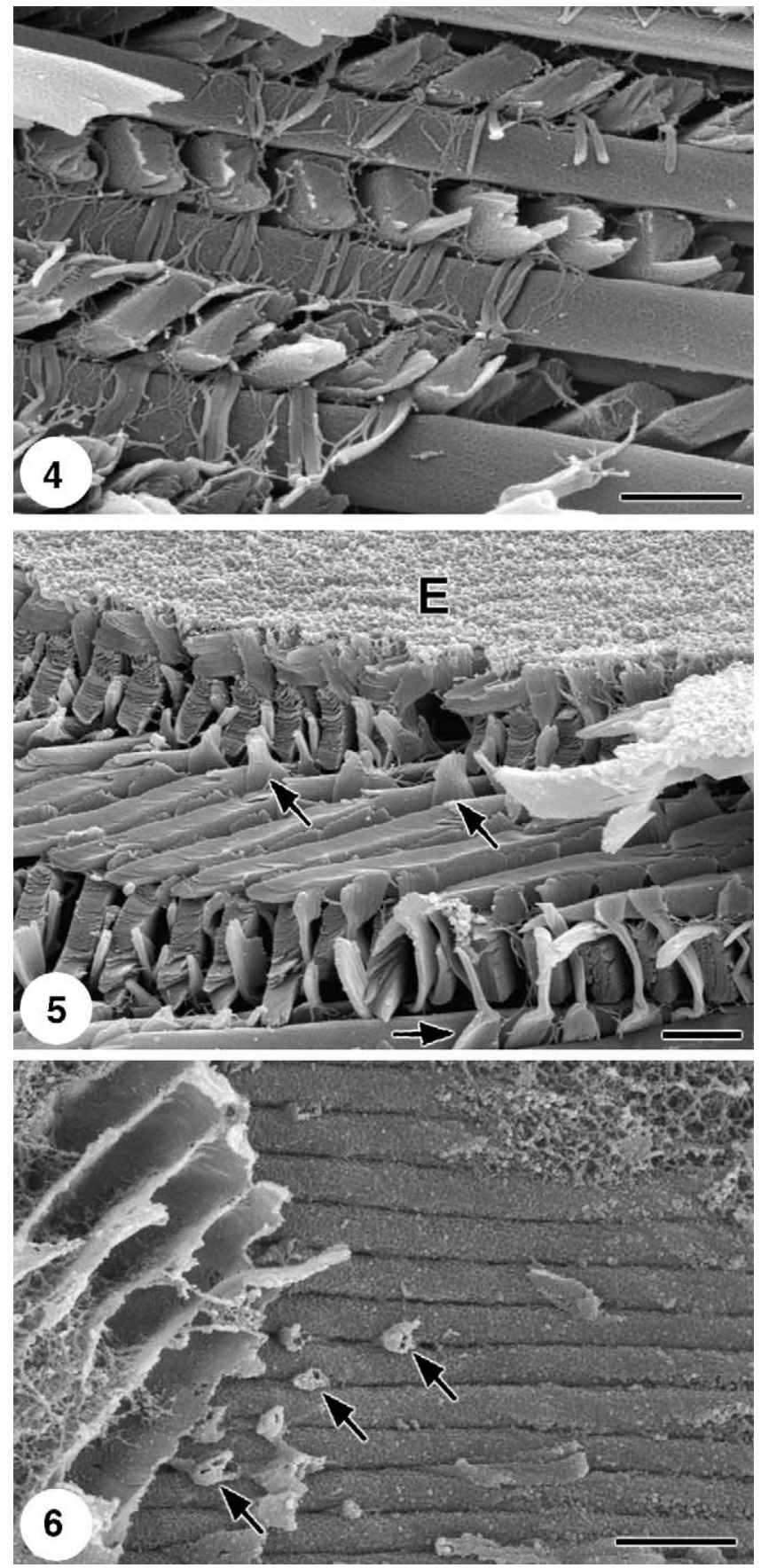

Fig. 4. SEM after $1 \mathrm{~N} \mathrm{NaOH}$ maceration. Cross-section of the cuticle. The large fibres are variously oriented in contiguous layers; ribbons of thin fibres run perpendicular in the narrow spaces between the large fibres. Thin fibres run perpendicular to bind the ribbons. Scale bar $1 \mu \mathrm{m}$. Fig. 5. SEM after $1 \mathrm{~N} \mathrm{NaOH}$ maceration. Tangential section of the cuticle. Some large fibres are transversally cut and some are tangentially cut: the fibres are composed by stacked planes of thin filaments. The cross-section clearly displays some radial fibres (arrows) running perpendicular to rejoin the external covering of the animal (E). Scale bar $1 \mu \mathrm{m}$. Fig. 6. SEM after short osmic maceration. Some slender tubular structures run perpendicular between the large fibres (arrows). Scale bar $1 \mu \mathrm{m}$.
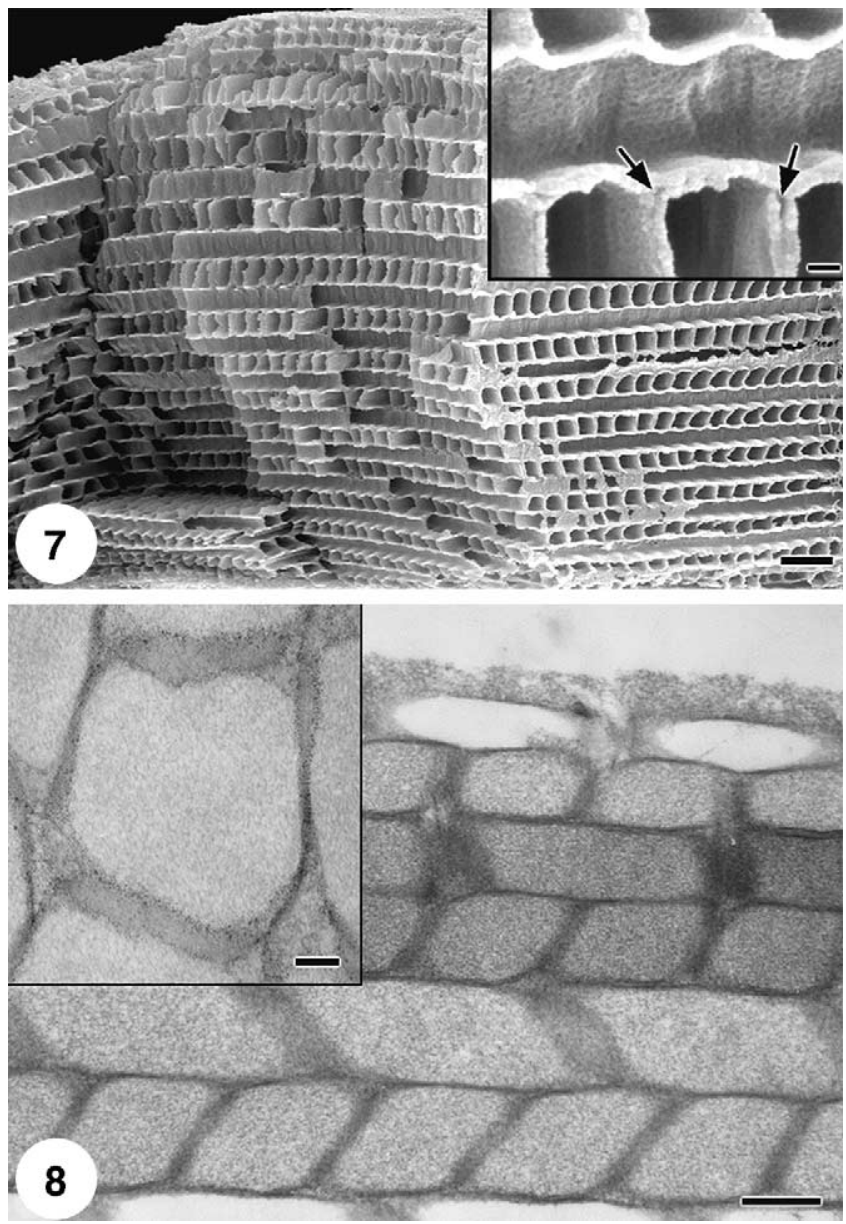

Fig. 7. SEM after prolonged osmic maceration, transverse section of the cuticle. Numerous layers of perifibrillar envelopes still maintain their spatial layout, with an inter-layer angle of $\sim 45^{\circ}$. Each shell is hollow and approximately rectangular in section. Scale bar $2 \mu \mathrm{m}$. Insert: Transverse section of three layers of the cuticle at higher magnification. Each perifibrillar covering is arranged side by side with the adjoining one; a narrow, empty space remains between the shells (arrows). The internal wall shows the regular net of hexagonal mesh. Scale bar $200 \mathrm{~nm}$. Fig. 8. TEM. Thiery reaction. The strong positivity (grey) is localised only on the perifibrillar covering. Scale bar $500 \mathrm{~nm}$. Insert: A magnification of a single perifibrillar shell. Scale bar $200 \mathrm{~nm}$.

appeared completely negative to the reaction, suggesting that the perifibrillar covering is polysaccharidic in nature (Fig. 8).

In atomic force micrographs the tissue architecture is well preserved and the pictures are entirely consistent with the scanning electron micrographs. Both the parallel large fibres and their perifibrillar covering are evident. Several radial thin fibres protrude from the crevices between the large fibres (Fig. 9).

At higher magnification, AFM resolved the perifibrillar network into an equally regular, double-stranded hexagonal mesh, where each hexagon is limited by a double filament. The ability of this technique to make direct measurements and virtual cross-sections allowed us to obtain some quantitative data: the sides of each hexagon measure $42 \mathrm{~nm}$ in 

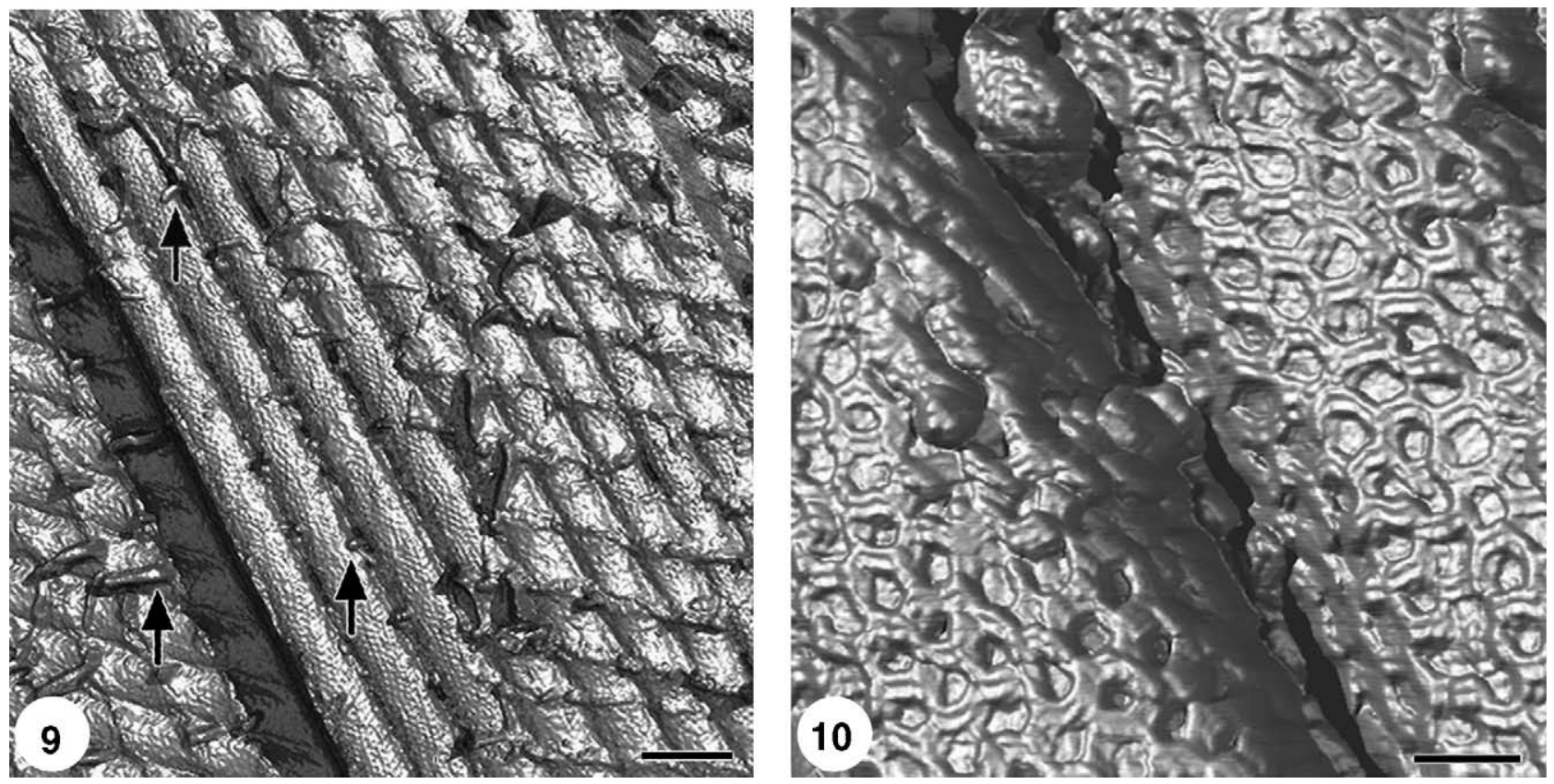

Fig. 9. AFM. The large parallel fibres and their covering are well evident. Radial thin fibres run between the large fibres (arrows). Scale bar $1 \mu \mathrm{m}$. Fig. 10. AFM. Detail of the covering of a single large fibre. The side of each hexagon is $42 \mathrm{~nm}$ long, $16 \mathrm{~nm}$ wide and protrudes $6 \mathrm{~nm}$ from the fibre surface. Scale bar $100 \mathrm{~nm}$.

length and $16 \mathrm{~nm}$ in thickness, and they protrude about $6 \mathrm{~nm}$ on the fibre surface (Fig. 10).

\section{Discussion}

The removal of the large fibres and the thin fibrils by osmic maceration confirms that they are essentially proteinaceous. However, the available data on the biochemical composition of the Nematomorph cuticle are not only very scarce, but also contradictory. While Swanson (1970) and Eakin and Brandenburger (1974) indicated a collagenous nature of the large fibres, Brivio et al. (2000) showed that large fibres remain unaffected by prolonged collagenase digestion, implicating the involvement of some structural insoluble protein, very stable and rich in dityrosine residues. Such proteins, called cuticlins, have been described in the cuticle of Nematodes, which are obviously closely related to Nematomorphs, but not much is known on Nematomorphs themselves. Although we only dealt with cuticle morphology, our failure to reveal any axial banding pattern on large fibrils corroborates the "non-collagen" hypothesis of Brivio et al. (2000).

In contrast, both the resistance of the perifibrillar hexagonal meshwork to osmic maceration and its positivity to the Thiery reaction both indicate a polysaccharidic structure. It is, of course, possible that other components are present, and it is noteworthy that Brivio et al. (2000) describe the mesh as sensitive to collagenase digestion. That the double-stranded structure of the meshwork revealed by AFM was never described points to a self-assembling supramolecular complex, reminiscent (from a morphological standpoint) of the "chicken wire" networks formed by type IV collagen, or of the regular triangular meshwork formed by its closely related type VIII in the Descemet membrane.

Finally, the thin, radial hollow shells observed in specimens treated with short osmic maceration are likely to be cytoplasmic extensions, derived from the epithelial cells which line the inner surface of the cuticle and run through all the layers of the cuticle to reach the epicuticle on the outer surface. The thin radial filaments grouped in narrow ribbons probably line the cytoplasmic extensions running through the cuticle (Eakin and Brandenburger, 1974).

The chemical stability, stiffness and spatial layout of these structures are certainly able to confer the strength and resilience the cuticle critically needs. Although the morphological approach disclosed new details of the cuticle components and their interactions, further studies are needed to typify and identify these structures from a chemical standpoint.

\section{References}

Bresciani, J., 1991. Nematomorpha. In: Harrison, F.W., Rupert, E.E. (Eds.), Microscopic Anatomy of Invertebrates. Wiley Liss, New York, pp. 197-218.

Brivio, M.F., De Eguileor, M., Grimaldi, A., Vigetti, D., Valvassori, R., Lanzavecchia, G., 2000. Structural and biochemical analysis of the parasite Gordius villoti (Nematomorpha, Gordiacea). Tissue Cell 32, 366-376.

Cham, S.M., Seymour, M.K., Hooper, D.J., 1983. Observation on a British hairworm, Parachordodes wolterstorffi (Nematomorpha, Gordioiidae). J. Zool. 199, 275-285. 
Eakin, R., Brandenburger, J.L., 1974. Ultrastructure features of a Gordian worm (Nematomorpha). J. Ultrastruct. Res. 46, 351-374.

Lanzavecchia, G., Valvassori, R., De Eguileor, M., Lanzavecchia Jr., P., 1979. Three-dimensional reconstruction of the contractile system of the Nematomorpha muscle fibres. J. Ultrastruct. Res. 66, 201-223.

Politz, S.M., Philipp, M., 1992. Caenorhabditis elegans as a model for parasitic nematode: a focus on the cuticle. Parasitol. Today 8, 6-12.
Swanson, C.J., 1970. An ultrastructural, biochemical and functional study of the body wall of Paragordius varius, and Gordius robustus Leidy. Ph.D. Dissertation, University of Illinois, Urbana, pp. 1157.

Thiery, J.P., 1967. Mise en evidence des polysaccharides sur coupes fines en microscopie electronique. J. Microscopie 6, 987-1017. 